\title{
Bandwidth Arrangement and Self-Healing Algorithm for Logical Mesh Connected Multi-Gigabit Ring Networks
}

\author{
M. Tomizawa, Y. Yamabayashi, N. Kawase, and Y. Kobayashi \\ NTT Optical Network Systems Laboratories \\ 1-2356 Take, Yokosuka, 238-03 Japan, Phone: +81-468-59-3065, \\ Fax:+81-468-59-3396, e-mail: tong@ntttsd.ntt.jp
}

\begin{abstract}
This paper proposes a novel bandwidth arrangement for an Add/Drop Multiplexer (ADM) that minimizes the line capacity required in bi-directional ring networks wherein the ADMs are connected logically in a mesh topology. The technique, characterized by timeslot/wavelength conversion, realizes uniform add/drop processing common to all ADMs, regardless of their location on the ring. A self-healing algorithm is proposed for the network, which offers better performance than existing algorithms for SONET rings in terms of protection line capacity, restoration time, and survivability against multiple failures. Delay issue caused by the self-healing function is also considered.
\end{abstract}

\section{Keywords}

ADM, logical-mesh ring, timeslot/wavelength conversion, dual ended shared path switching

\section{INTRODUCTION}

It is expected that Metropolitan Area and Wide Area Networks (MANs and WANs) will have to handle many kinds of Local Area Networks (LANs) providing different services with widely differing bit-rates and differing protocols, together with ordinary sub-networks. In such a situation, multiplexing various signals into a single network is the only way to achieve reasonable cost. Obviously, network survivability becomes more important as the number of multiplexed channels and the transmission line bit-rate increase (Wu, 1992).

A ring structure is a physical network topology and offers both effective cost and survivability (Wu, 1990, To, 1991) for MAN or WAN applications. The ring achieves lower costs due to its reduced conduit space requirements or low number of links, and higher survivability through its self-healing function (Wu, 1990). However, the ring requires larger line capacity since fewer routes are available to support crossing traffic. To date, Hill has reported that the ring topology requires the maximum number of wavelengths among the optical path network topologies that use Wavelength Division Multiplexing (WDM) (Hill, 1988). In an interesting development, Elrefaie has introduced a wavelength assignment table 
that minimizes the number of wavelengths for full-mesh connected bi-directional rings for systems with fewer than 11 nodes (Elrefaie, 1993). However, the wavelength allocation table is determined by a central control node, which leads to a centralized control environment and so exposes the network to the complete system down with control node failure. Moreover, general assignment, for an arbitrary number of nodes, that optimizes line capacity is an outstanding problem.

This paper proposes a novel bandwidth grooming scheme for Add/Drop Multiplexers (ADMs) that minimizes the line capacity required by the logical mesh topology. The proposal is advantageous due to its uniform processing scheme common to all ADMs in the ring, while releasing us from a bandwidth allocation problem. Moreover, the novel selfhealing algorithm that best suits the ring is proposed (Tomizawa, 1994). It offers better performance than existing rings using the Synchronous Optical NETwork (SONET) protocol, in terms of protection capacity, restoration ratio, and survivability against multiple failures.

First, the multiplexing structure that will be employed in future MANs or WANs is discussed. Section 3 considers connectivity requirements in future networks. This leads us to a full-mesh connected ring for a distributed control environment. Section 4 describes a novel bandwidth grooming technique for a fully connected mesh topology. The scheme is examined in both time and frequency domains for application to rings using either Time Division Multiplexing (TDM) or WDM. Section 5 proposes a self-healing algorithm that can be described as dual-ended shared path switching. Section 6 estimates the performance of the proposed algorithm. Section 7 considers transmission delay taking self-healing functions into account, which limits the network scale. This paper concludes in section 8 . Section 9 includes acknowledgments, and 10 is the appendix.

\section{MULTIPLEXING STRUCTURE}

LANs are required to achieve gigabit throughputs for connecting several computer terminals driven by powerful processors. In that, technologies for constituting gigabit LANs have been actively studied and developed. Examples include High Performance Parallel Interface (HIPPI), Fiber Distributed Dual Interface (FDDI), Fiber-Channel (FC), or Asynchronous Transfer Mode (ATM), each of which is fascinating for gigabit LANs. On the other hand, MANs or WANs should handle data traffic between these LANs of differing bit-rates and of differing protocols, video broadcasting traffic, even telephone traffic. Hence in MANs or WANs, a unified or standardized protocol is needed, by which signals of differing bit-rate are multiplexed and transmitted to the network. ATM protocol seems to be a solution but it has several problems: 1) The trade-off between Quality of Service (QoS) and the ATM node buffer size (Fischer, 1994). Small buffers increase the cell loss rate, while large buffers increase cell delay. 2) The problem of queue and jitter (Aoyama, 1992). Queueing time and cell-delay fluctuation increase when many large nodes are attached to the network. 3) The flow control problem. Even if a flow control function based on whether mean or peak cell rate is realized, cell loss probability cannot be completely excluded. Moreover, closed-loop reactive flow control based on Available Bit-Rate (ABR) is not feasible in a large network because of its long feed-back time (Fischer, 1994). 4) The traffic model problem (Partridge, 1994). Because burst cell traffic cannot be described by existing statistical traffic models, no 


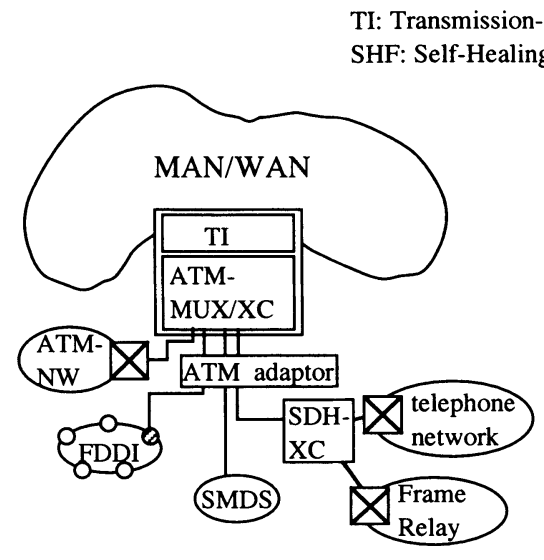

(a) ATM Multiplexing

Figure 1 Multiplexing structure

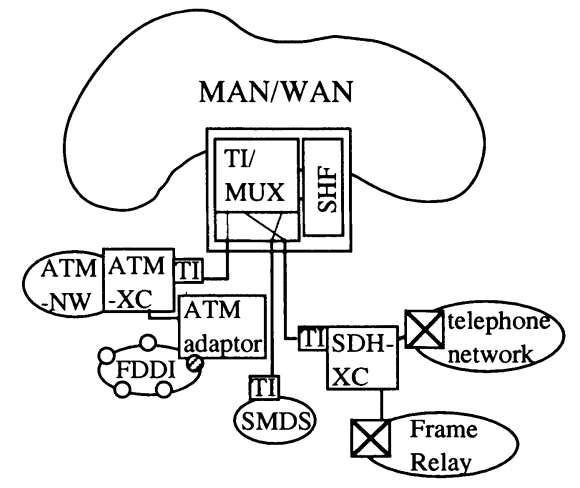

(b) Physical Multiplexing

one can exactly predict the cell loss probability. These problems may originate in trying to use statistical multiplexing to increase transmission resource efficiency utilization.

There are two multiplexing structures that can allow MAN/WAN nodes to be connected to gigabit LANs. How to realize the node functions, e.g. termination, multiplexing and routing, determines the multiplexing structure. The first is an ATM multiplexing after solving all the problems mentioned above, where all signals are encapsulated into ATM cells, every routing is executed cell by cell, multiplexing in a statistical manner, and then signals are transmitted to the network after being terminated in Transmission-line Interface (TI) format, for example a Synchronous Digital Hierarchy (SDH) frame. The second is a physical multiplexing, where all functions can be done in TI format at a physical layer. Map different kinds of signals into TI, multiplexing proceeds in a fixed manner. The multiplexing structures are shown in Figure 1(a), (b). In Figure 1(a), the MAN/WAN node is equipped with an ATM-Cross-Connect system (ATM-XC) to which ATM-LAN, Frame Relay network, Switched Multi-megabit Data Services (SMDS), and SDH network are connected as sub-networks. The signals in these sub-networks are segmented into ATM cells at the ATM adaptor, and their routes are determined by setting their cell headers (Virtual Path Identifier: VPI), and are arranged at ATM-XC. They are mapped into TI at the TI adaptor. On the other hand, in Figure 1(b), these sub-networks are connected to low-speed ports of a multiplexer (MUX) after the signals are mapped into TI. Each MUX low-speed port is connected to that of the opposite node so that each sub-network can communicate with the same kind of sub-network.

It should be noted that network administration is quite different between two structures. ATM multiplexing requires an OAM-cell administration, while physical multiplexing provides a path administration. We adopt physical multiplexing because of the following two reasons: it offers an excellent quality of signal monitoring in network administration, and is free from the flow comtrol problem which is more serious as the transmission line-rate getting higher: several tens of giga-bit/s. However, it offers inefficient usage of transmission resources. We adopt physical multiplexing at the expense of efficiency of circuit 
accomodation into transmission line, but which makes it possible to achieve line bit-rates from $10 \mathrm{Gbit} / \mathrm{s}$ to $1 \mathrm{Tbit} / \mathrm{s}$ by using optical technologies.

Multiplexing technologies for very high-speed optical systems can be categorized into two types. First is SDH or SONET using TDM scheme. SDH is based on STM-1 of 156 Mbit/s and consists of other higher rates such as STM-4 of $622 \mathrm{Mbit} / \mathrm{s}$ and STM-16 of 2.4 Gbit/s. These transmission rates have been standardized by International Telecommunications Union-Telecommunication (ITU-T). NTT is developing an STM-64 system which is the $10 \mathrm{Gbit} / \mathrm{s}$ version of SDH (Kobayashi, 1994), and ITU-T is discussing its standardization. At present, SDH is the most promising technology for multi giga-bit/s multiplexing and transmission.

Another candidate is WDM, by which the broadband characteristics of optical fiber can be fully utilized. A European WDM network trial commenced in 1992 (Hill, 1993). Several proposals have been made for WDM packet networks such as the single-hop or multi-hop scheme. However, in this paper, we restrict ourselves Optical Path configuration (Sato, 1994) in which wavelengths are assigned to connected paths between nodes in the MAN/ WAN. High transmission capacity, exceeding that of SDH, is obtained by the summation of the bit-rate of each wavelength.

Two architectural issues arise in network nodes as shown in Figure 1(b). 1) How to decide which route a path takes for a connection, 2) how to restore network failures. Using the ring network architecture, the node configuration is simplified. A path route is simply one of just two directions, clockwise or counterclockwise, furthermore network failure can be restored automatically: the self-healing function is adopted. This paper proposes a bandwidth arrangement scheme to assure path connection and also a self-healing algorithm for failure restoration.

So far, there are ring networks based on SDH/SONET, called SONET rings (Wu, 1994), that were designed to support mainly point-to-point traffic. However, the transmission speed of SONET rings is limited to STM-16/OC-48 and below. Although ring networks beyond $10 \mathrm{Gbit} / \mathrm{s}$ are necessary from the viewpoint of service economy (Wasem, 1992), they do not exist. WDM ring networks have been proposed as described in section 1, however, further studies are still needed because the architecture and devices strongly depend on further progress in optical technologies. It is important to establish a node function that is applicable to both SDH (TDM) and WDM. This will suggest a common Operation System (OpS) that ignores the physical differences between multiplexing schemes. In other words, when extending throughput, SDH to WDM, the additional changes should be suppressed as much as possible.

The next section considers connectivity, an important issue when multiplexing various kinds of services into a single network.

\section{CONNECTIVITY CONSIDERATION}

The future MAN/WAN must be flexible enough to achieve any level of connectivity as various kinds of services will be supplied through many sub-networks (McEachern, 1992). Thus, connectivity management will be unavoidable. In a network supporting only point-topoint traffic, the operation system drives the XCs forming the path connections. However, it is difficult to support multi-cast services in the same manner: XCs should be able to support 


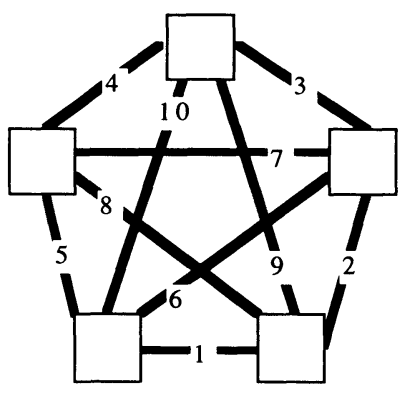

(a) Logical connection

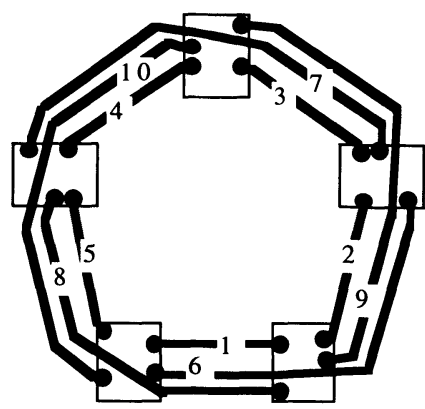

(b) Physical connection

Figure 2 Logical mesh connection on ring network

any level of connectivity, and this requires a tremendous throughput. On the other hand, power users will be able to or even eager to determine the level of connectivity by themselves. For basic users, however, the network provider should control the connectivity. In such a complex situation, the network can be simply configured by logically connecting all nodes in a mesh topology, regardless of individual communication activity needs. The highest connectivity is realized with the full-mesh demand pattern that is expected in the later stages of broadband evolution (Elrefaie, 1993). Lower connectivity demands (star, point-to-point, etc.), can be satisfied by using a portion of the mesh. In networks wherein the nodes are always connected in a logical mesh, connectivity management is distributed among the nodes regardless whether they are owned by the user or the network provider. This leads to a distributed network control environment which increases network survivability against control node failures. We restrict ourselves hereafter to a logical-mesh connected ring network. In a meshed ring, each node is assumed to occupy a fixed share of the total bandwidth all the time as in SONET rings or WDM rings, as is true for much of the conventional Time Division Multiple Access (TDMA) networks. Although this type of ring network suffers no traffic-dependent delay or loss, it is less efficient in this usage of the transmission capacity. Simple protocols of this type are suitable for optical transmission as long as it appears to have "unlimited" capacity (Kataoka, 1992, Kawanishi, 1993). However, since future services need extremely high bit-rates, the efficient usage of capacity will be important in rings of several tens of giga bit/s. We assume a bi-directional ring network with the logical mesh topology (see Figure 2) with the goal of maximizing the efficiency of the working line. In Figure 2, the number of nodes (ADMs) is 5 for simplicity. Figure 2 (a) shows the logical connection of paths. The internal paths (paths numbered 6 to 10 in the figure) are replaced with double length paths that follow the periphery of the ring as depicted in Figure 2 (b), where the filled circles represent adding or dropping points. When the minimum capacity is satisfied, each line has the same number of paths, in this case 3. Each path takes the shortest route independent of direction. For an arbitrary number of nodes, the minimum line capacity of a bi-directional ring is represented as (Elrefaie, 1993):

$\mathrm{N}$ : even number of paths per link $=\left[\frac{N^{2}}{8}\right]$,

$\mathrm{N}$ : odd number of paths per link $=\frac{N^{2}-1}{8}$, 
Table 1 Switch Scale Comparison

\begin{tabular}{lcc}
\hline \multirow{2}{*}{ Equipment } & \multicolumn{2}{c}{ Switch scale $O(\mathrm{x})$} \\
& matrix switch & Benes switch \\
\hline \hline XC in hub(star) network & $\{\mathrm{N}(\mathrm{N}-1)\}^{2}$ & $\mathrm{~N}(\mathrm{~N}-1) \log _{2}\{\mathrm{~N}(\mathrm{~N}-1)\}$ \\
ADM in ring network & $2\left\{\left(\mathrm{~N}^{2}-1\right) / 8\right\}^{2}$ & $2\left(\mathrm{~N}^{2}-1\right) / 8 \log _{2}\left\{\left(\mathrm{~N}^{2}-1\right) / 8\right\}$ \\
\hline
\end{tabular}

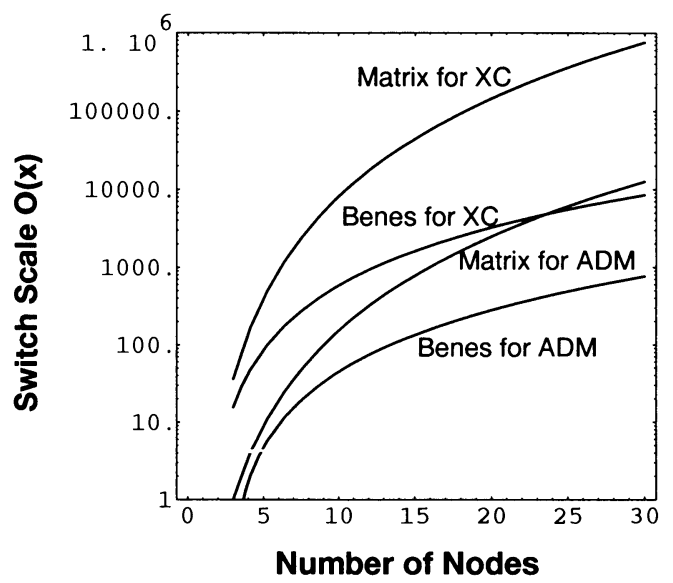

Figure 3 Swich Scale Comparison

where $\mathrm{N}$ is the number of nodes, and [ ] means a Gauss symbol that rounds off non-integer values.

If the minimum capacity is realized, the $\mathrm{ADM}$ in the efficient ring requires only small scale switching equipment, and so is superior to the $\mathrm{XC}$ in hub or star network topologies which is an extension of point-to-point transmission systems. Only the central node executes the switching function in hub (star) topology, while in ring, control is distributed among all nodes which obviates the risk of complete system down after a control node failure. To calculate switch size, some assumptions must be made. Each mesh connected path occupies the same capacity. The switch is assumed to be a matrix type or Benes type. The ADM switch is able to add/drop arbitrary paths from/to the multiplexed signal stream. Link capacity required in the full mesh connection is $\mathrm{N}-1$ for hub (star) (Hill, 1988), and $\left(\mathrm{N}^{2}-1\right) / 8$ for this bi-directional ring. The number of input or output ports connected to the switch is $\mathrm{N}(\mathrm{N}-$ 1) for the hub, $\left(\mathrm{N}^{2}-1\right) / 4$ for the ring. For an arbitrary number of ports $\mathrm{K}$, switch scale is $\mathrm{o}\left(\mathrm{K}^{2}\right)$ for the matrix switch and $\mathrm{o}\left(\mathrm{K} \log _{2} \mathrm{~K}\right)$ for the Benes switch as shown in Table 1. Figure 3 compares the switch size of the $\mathrm{ADM}$ in the ring and the $\mathrm{XC}$ in a hub. As in the figure, ADM switch size for the matrix type is one or two orders smaller than that of the XC. Figure 3 also shows the difference in switch size for the Benes type switch, in which ADM achieves a size reduction of one order. 


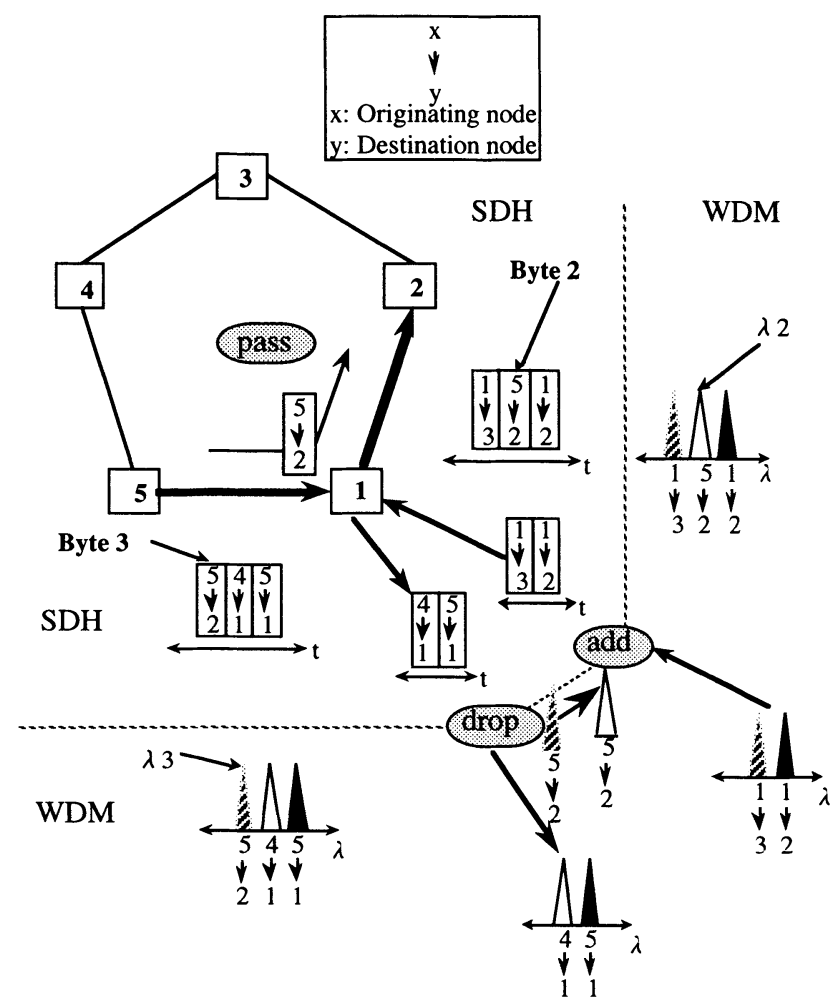

Figure 4 Bandwidth arrangement for SDH/WDM ring network

\section{BANDWIDTH ARRANGEMENT}

Here we propose a novel bandwidth arrangement applicable to both SDH and WDM that realizes the maximum line usage. Figure 4 shows, for the case of 5 nodes, details of the reuse of bandwidths in SDH and WDM, where each node is an ADM. Each byte is represented as a box in which the originating node number and destination node number are given by the upper and lower figures, respectively. Considering the byte-interleaving nature of SDH (Yamabayashi, 1993), each box has a time length of 8 bits. For the bit-interleaving TDM scheme, each box will be a timeslot, though this paper restricts itself to SDH. Three bytes are required for one cycle on the multiplexed signal stream for mesh connectivity, while lower connectivity demand patterns require fewer bytes. Since the network should support either type of demand pattern, the bandwidth grooming scheme is optimized for mesh connections. In Figure 4, we focus on node \#1, and only counterclockwise signals are shown. It should be noted that the remaining paths mapped to node \#1 run clockwise. The first byte is used for the path from the nearest neighbor (node \#5) to node \#1 (which is 


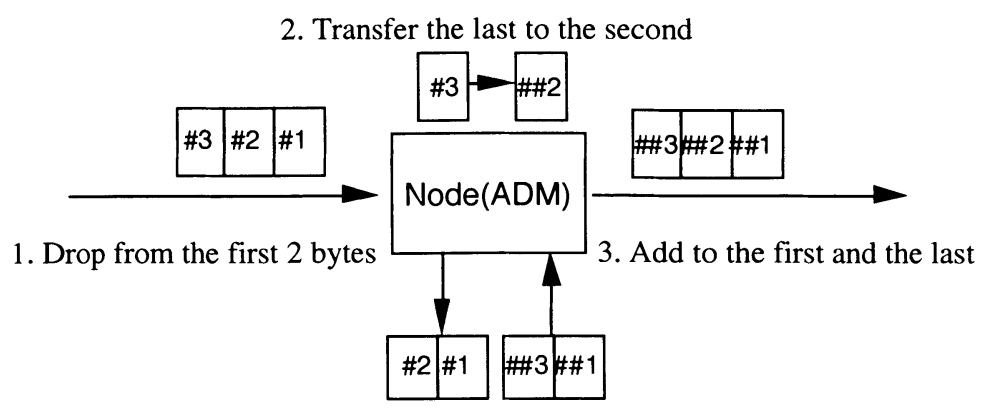

Figure 5 Universal processing for each ADM

expressed by $5 \rightarrow 1$ hereafter), and the second is for the path from the second nearest neighbor node \#4 to \#1(4 $\rightarrow 1)$, the last one is for $5 \rightarrow 2$. Node \#1 processes signals as follows: node \#1 drops signals indicated by the first two bytes, and then adds signals for paths $1 \rightarrow 2$ and $1 \rightarrow 3$ to the first and last bytes, respectively. Therefore, the output signal from node \#1 consists of $1 \rightarrow 2,5 \rightarrow 2$, and $1 \rightarrow 3$. At node \#1, the byte of the pass-through signal is moved from the last byte to the second. The proposed procedure makes it possible for every node to handle their signals in exactly the same manner as depicted in Figure 5. That is, 1. drop the first two bytes, 2 . transfer the last to the second, and 3. add to the first and the last positions.

In the proposed method, the generality of add/drop processing is valid for any arbitrary number of nodes. A detailed description for arbitrary node number will be reported elsewhere (Tomizawa, to be submitted). Figure 6 shows input and output bytes at node \#1 for the case of 9 nodes. In this figure, every node drops four bytes from the top and adds signals to the first, fifth, eighth and tenth bytes. Signal $9 \rightarrow 4$ is transferred from byte 10 to byte 9 , $8 \rightarrow 3$ moves from 9 to 7 , and so on.

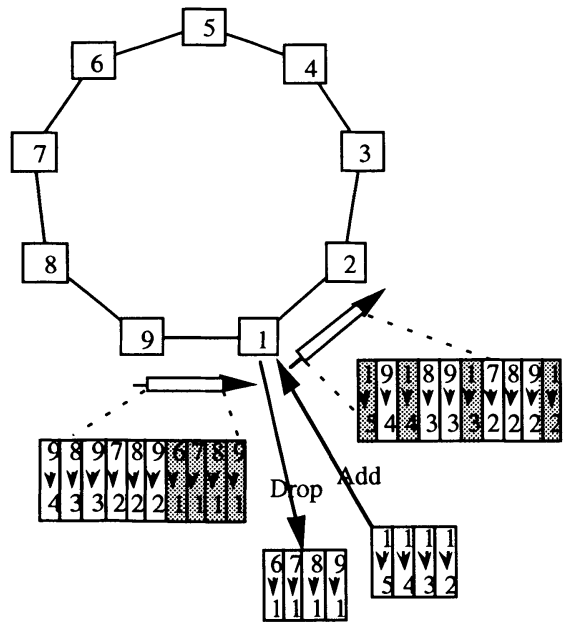

Figure 6 Bandwidth Arrangement, 9 nodes

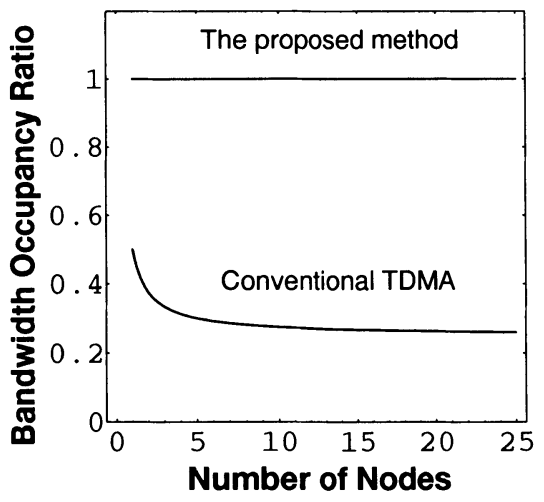

Figure 7 Bandwidth occupancy ratio 
In a bi-directional ring network using conventional TDMA, the absolute position of timeslots from one node to another is fixed. Hence, whether a path takes a shorter route or longer route, its timeslot must be retained in every link. TDMA requires the capacity of all paths in each link to be $\mathrm{N}(\mathrm{N}-1) / 2$. Here, we define the bandwidth occupancy ratio as the ratio of occupied capacity over required capacity. Bandwidth occupancy ratio is depicted in Figure 7 as a function of the number of nodes. With the proposed method, the bandwidth occupancy ratio is independent of the number of nodes and is always 1 , the maximum value. On the other hand, the TDMA method has a maximum bandwidth occupancy ratio of $1 / 2$ at $\mathrm{N}=2$, and the ratio decreases as the number of nodes increases to the asymptotic value of $1 / 4$. Thus, the proposed method yields obviously superior bandwidth efficiency. Furthermore, TDMA and some SONET rings require the center control node to deliver a large pathtimeslot table to each ADM, and an amount of memory is required in each node. The proposed scheme is hardware oriented, in this sense.

WDM networks basically follow the byte exchange method described above: wavelengths replace time-positions in each byte. Figure 4 also depicts the methodology of reusing wavelengths. Wavelengths are sketched in spectral form. Paths mapped to node \#1 are: path $5 \rightarrow 1$ to wavelength $\lambda_{1}$, path $4 \rightarrow 1$ to $\lambda_{2}$, and path $5 \rightarrow 2$ to $\lambda_{3}$. Node \#1 drops $\lambda_{1}$ and $\lambda_{2}$ and adds path $1 \rightarrow 2$ on $\lambda_{1}$ and path $1 \rightarrow 3$ on $\lambda_{3}$. Concurrently, the wavelength of path $5 \rightarrow 2$ is converted from $\lambda_{3}$ to $\lambda_{2}$. With 28 nodes, the number of wavelengths required in this method is 98 which is possible given today's technology (Nosu, 1993). If a network has more than 28 nodes, adding multiple fibers will be a cost effective solution although it depends upon the maturity of optical technologies.

The uniformity of add/drop processing remains valid for WDM without change. In Elrefaie's wavelength assignment method (Elrefaie, 1993), the wavelength treatment of one node differs from that of the others. Since wavelengths dropped, added or passed-through in each node are not the same, hardware (for example, optical filters) must be customized. Otherwise, the hardware should be tunable or programmed from the central node as are existing XCs. In the proposed method, which realizes uniform processing, each ADM does not require tunable or programmable functions. This simplicity is suitable for implementing large capacity networks using either optical WDM or digital SDH whose hardware constraints are severe. The method has the potential of significant cost reductions because the nodes can be mass-produced as replicas. In addition, the maturation of optical technologies will realize low cost wavelength converters. Along with these points, our network with wavelength conversions has two more advantages as mentioned by Sato et al. (Sato, 1994). First, path-wavelength administration can be controlled in a distributed manner. This is more suitable for the distributed control environment of the mesh-connected ring as described in section 3 than the centralized management required in Elrefaie's method. Second, wavelength preciseness is required only between the link terminating nodes, which results in relaxed requirements for optical devices.

\section{SELF-HEALING ALGORITHM}

This section proposes a self-healing algorithm applicable to the byte/wavelength convertible ring network described in the preceding section. Because the ring network is bi-directional, each link requires two more fibers for the protection ring, hence, each link needs 4 fibers. In 


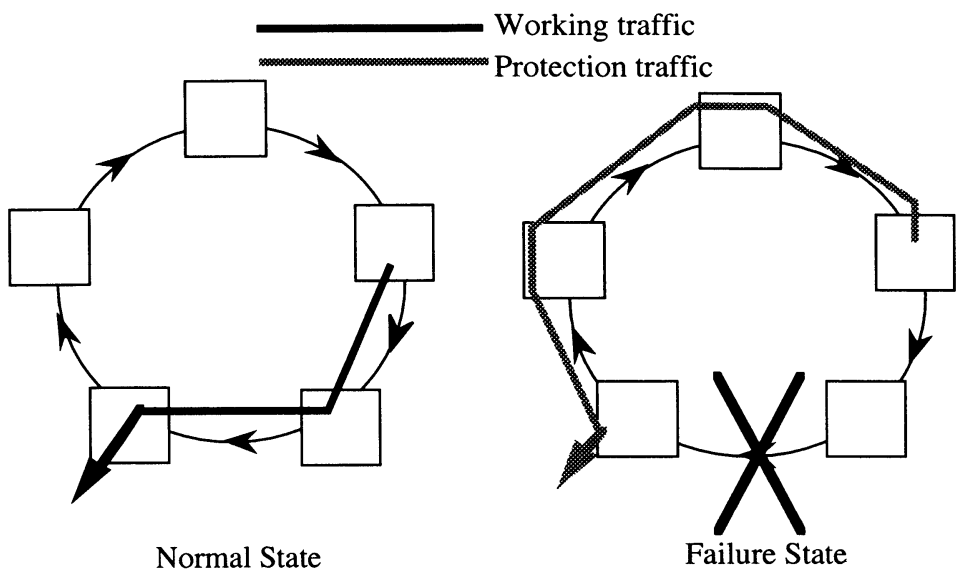

Figure 8 Self-Healing Algorithm

our ring network, the time-position or wavelength occupied by one path changes as it traverses each working line. Each path is assured of logical connection between nodes, and does not participate into the assignment of time-position/wavelength in the physical working line. In addition, the distributed network control environment is required as described in Section 3. Therefore, it is quite natural to expect each end node to perform restoration switching, which leads to a self-healing algorithm on path switching, where path restoration is triggered by a path alarm after line failure. The proposed algorithm employs dual-ended shared path switching for the efficient usage of protection line capacity. By using this algorithm, protection line capacity is utilized only by the failed link, because only "failure paths" are switched to the protection line, while in the no-failure state, the protection ring has no traffic or only extra traffic (Bellcore Technical Reference, 1991). Therefore, the capacity of the protection line equals that of the working line. Figure 8 shows a normal state and a failure state, where black line represents working path while gray one protection path. ADM

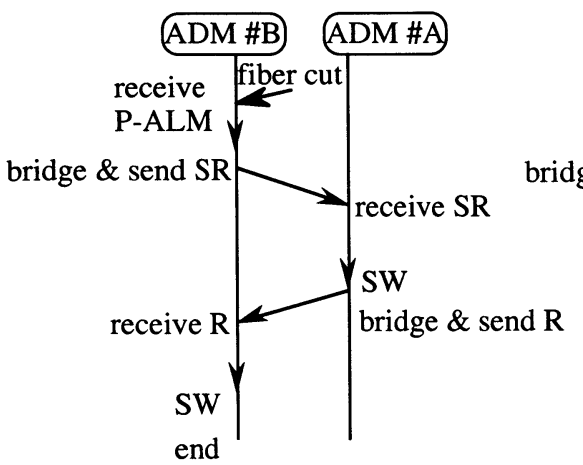

(a) In case of a fiber (from A to B) cut

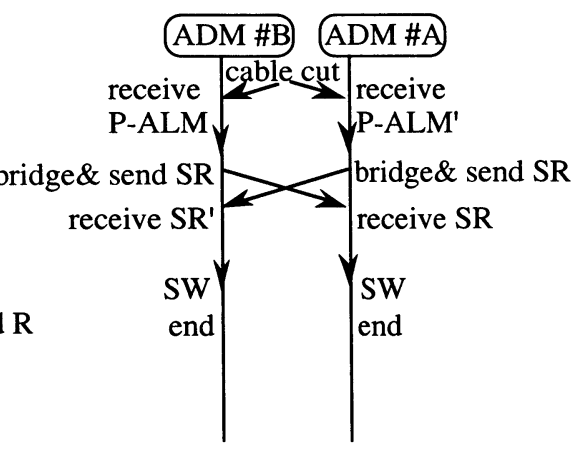

(b) In case of a cable cut

Figure 9 Switching Control Scheme of the Proposed algorithm 
of receiver side detects the alarms issued for the path. The path is restored by establishing counter rotating routes in the protection ring by dual-ended path switching. Switchings are executed not only at the receiver side but also at the transmisster side. It should be noted that the assignment of failed paths, in terms of bytes/wavelengths, is determined only by the location of the failure.

Figure 9 depicts the switching control scheme of this algorithm, where only end nodes are shown. If a fiber is cut as shown in Figure 9 (a), ADM \#B receives the path alarm (PALM), and transmits the same signals as the failure path (bridges) through the protection ring together with the switch request signal (SR) including the node ID number to \#A. When \#A detects SR from \#B, \#A switches the path, and bridges the path in the opposite direction with the returning signal (R) including ID to \#B through the protection ring, after switching is complete. Node \#B switches the path when it receives $\mathrm{R}$ from \#A. In the case of a cable failure somewhere between ADM \#A and \#B as shown in Figure 9 (b), either node \#A or \#B detects P-ALM. Each node bridges and sends SR to the opposite node through the protection ring. Detection of SR from the opposite node triggers switching of the path connected to the originating node.

In SDH, each control signal will occupy 1 byte somewhere in the Path Overhead (POH), while in WDM, controlling protocols remain to be developed. The proposed algorithm is easily extended to restoration switching without bit-loss (hitless switching) by implementing a phase adjusting circuit in each ADM. During ordered switching, for example adding or deleting nodes or construction work, even single bit-loss can be avoided.

\section{ALGORITHM PERFORMANCE}

The algorithm is compared with ordinary solutions such as SONET path switching and SONET loopback switching (ITU, 1994) using three parameters: protection line capacity, restoration time, and survivability. SONET path switching is applied to uni-directional

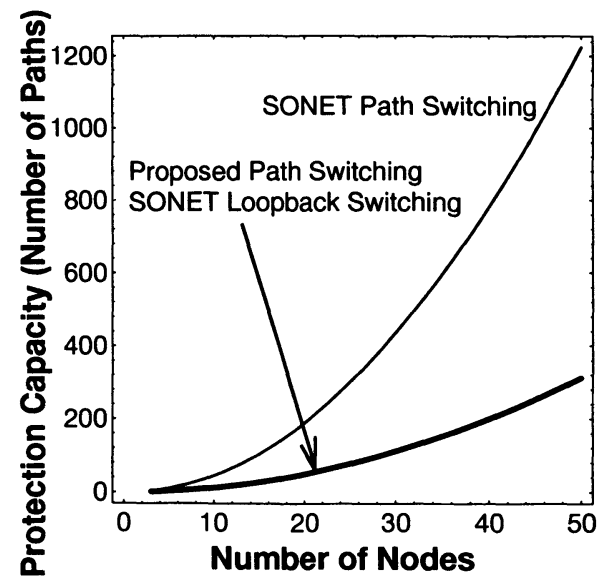

Figure 10 Protection line capacity required in each switching scheme

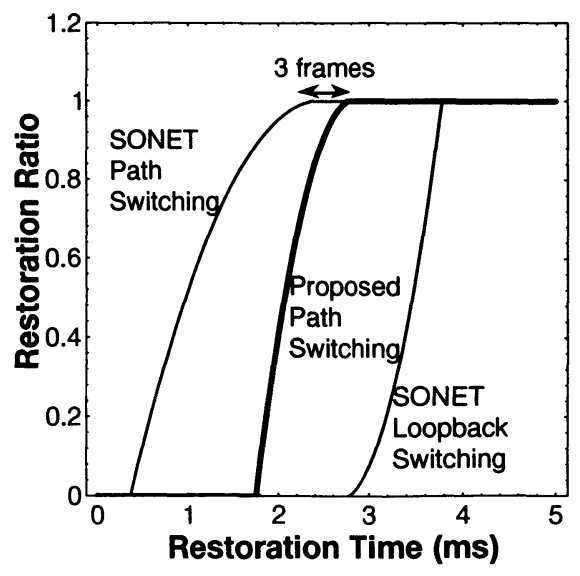

Figure 11 Restoration Ratio as a function of restoration time 
rings, where each node transmits the same signal through both working and protection rings simultaneously, and switches the receiver side when a failure occurs. On the other hand, SONET loopback switching is mainly applied to bi-directional rings. Only the nodes adjacent to the failed fiber section switch. To restoring a failure, it connects the working ring to the protection ring at the nodes adjacent to the failure to form a new loop.

A comparison of the line capacity (number of paths) required by the protection ring is depicted in Figure 10. The protection capacity required by the proposed configuration equals that of SONET loopback switching, i.e., about $25 \%$ of that of SONET path switching. Because SONET path switching is applied to uni-directional rings with $1+1$ dedicated single-ended switching, protection capacity is required for all paths in every link: $N(N-1) / 2$.

Figure 11 shows the restoration ratio as a function of restoration time against a cable cut. The restoration ratio is defined as the ratio of the number of restored paths to that of failed paths. The calculation uses the following parameters; number of nodes is 16 , the delay time per passing-through node is $0.010 \mathrm{~ms}$, the delay in each adding/dropping node is $0.005 \mathrm{~ms}$, node distance per link is a uniform $25 \mathrm{~km}$, and guard time for detecting the control signal is 3 frames long for each command. The proposed algorithm offers better performance than SONET loopback switching. The reason is that the latter forces the restoration path to passthrough the nodes adjacent to the failure. This often causes loopback which increases restoration time. The time difference increases in a larger scale network, since it depends on the propagation delay of restored signals in the ring. SONET path switching shows better performance than the proposed scheme because switching requests are not required for dedicated switching at the expense of increased protection capacity. Detailed calculations of these three algorithms are described in the Appendix.

We estimate the survivability of the proposed algorithm against multiple failures as follows. Survivability is defined as the ratio of surviving paths after failure to all paths. In the case of a single failure, perfect restoration is assured by all three algorithms. However, with multiple failures, survivability depends on the failure positions. Hence, we calculated the statistical average of survivability for all possible failure positions for several failure

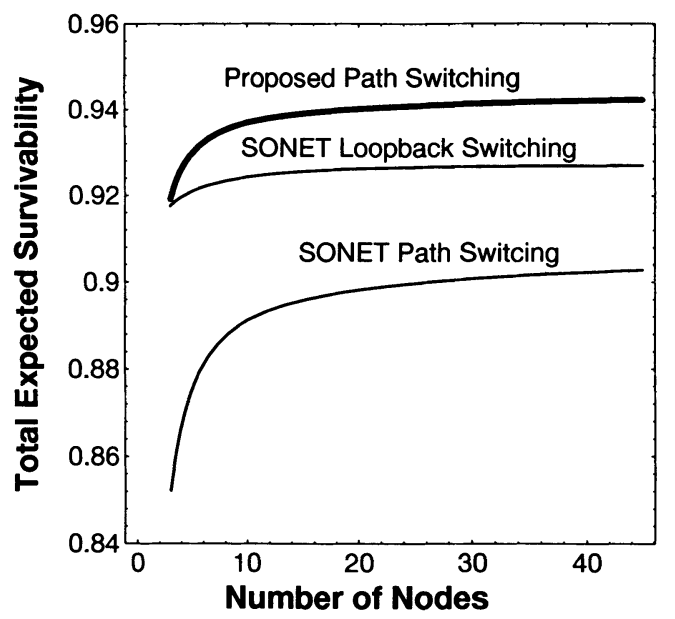

Figure 12 Total expected survivability 
Table 2 Comparison of performances of self-healing algorithms

\begin{tabular}{|c|c|c|c|}
\hline Parameters & SONET Loopback & SONET Path & Proposed Path \\
\hline Shared/Dedicated & Shared & Dedicated & Shared \\
\hline Switch back function & required & no required & required \\
\hline Single/Dual ended switch & dual & single & dual \\
\hline Protection capacity & 1 & $\simeq 4$ & 1 \\
\hline Propagation delay for restoration & long & short & middle \\
\hline Survivability against multiple failures & medium & weak & strong \\
\hline Switching protocol & APS & no & proposed \\
\hline $\begin{array}{l}\text { Extention to hitless switching } \\
\text { (buffer size) }\end{array}$ & difficult & easy & easy \\
\hline
\end{tabular}

number. Survivability was determined by assuming that each failure occurs independently (Liew, 1994): the probability function of failure follows a Poisson distribution with the most frequent number of failures taken to be 1 . The overall survivability values are plotted in Figure 12 as a function of the number of nodes. The mathematical model and detail calculations will be reported elsewhere. The proposed switching algorithm offers the best survivability. The proposed switching scheme will protect the paths more than the other schemes. This scheme has shorter routes in the working ring than SONET path switching, while it generates shorter restoration routes than SONET loopback switching. Because of this, the proposed switching scheme provides less chance for the paths to suffer subsequent failure. The differences among total expected values seem to be small because of Poisson distribution function for probability of failure happenings. However, once multiple failures occur, they cause significant differences of survivability among three algorithms. In the figure, the total expected survivability tends to increase with the number of nodes. The reason originates in the definition of survivability: with a large number of nodes, single failure is less crucial relatively to that with a small number of nodes. Survivability differs from reliability which can be defined as the probability of preventing failure.

The self-healing algorithms are compared in Table 2, which shows eight parameters: shared/dedicated, requirement of switch-back functions, single/dual-ended, protection capacity, restoration time, survivability against multiple failures, necessity of communication protocols, and extension to hitless switching. The proposed algorithm shows better performance attributes than existing self-healing algorithms.

\section{DELAY CONSIDERATION}

If the proposed ring network includes the self-healing function, protection traffic experiences longer delay than the working one. The transmission delay in digital networks has been fixed by ITU-T (CCITT, 1988). Normally, services are provided to users through the working channel which satisfies the specification. Even after a failure, services should be supplied in compliance with the specification regardless of whether they are transmitted through the protection line. In this section, we estimate the upper limit of the network scale given 
that the protection delay equals the specification of the working ring. A comparison with loopback switching is also shown. Because the uni-directional ring has a different specification, SONET path switching is omitted from consideration. The condition used in the estimation is as follows.

1) The specified delay between digital exchanges, which is called Maximum Affordable Delay (MAD) in this section, is

$\mathrm{MAD}=3.0(\mathrm{~ms})+$ propagation time.

Therefore, MAD depends upon the transmission distance.

2) MAD in a ring network is assumed to be the delay for the longest path in the working line. All node distances are equal $\mathrm{L}(\mathrm{km})$ for simplicity. In bi-directional rings, MAD becomes,

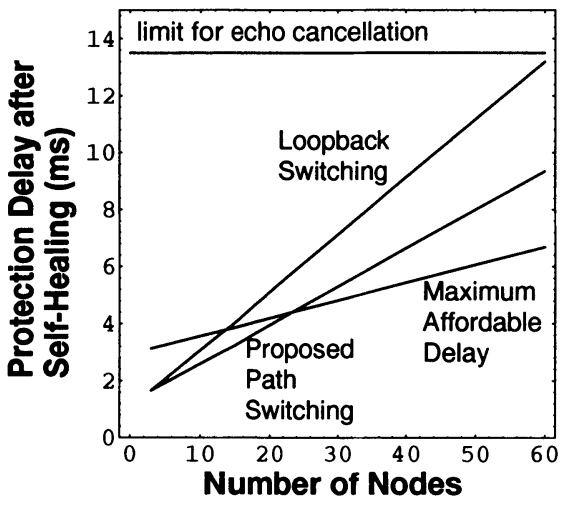

(a): Node distance $25 \mathrm{~km}$

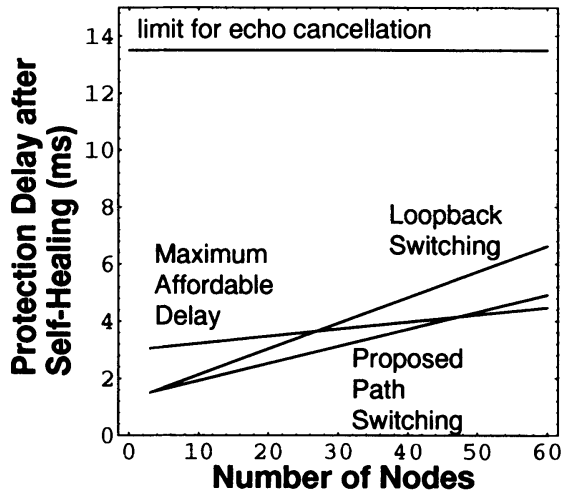

(b): Node distance $10 \mathrm{~km}$

Figure 13 Protection delay for self-healing switching schemes

Table 3 Network scale estimation from delay consideration

\begin{tabular}{llc}
\hline Parameters & \multicolumn{1}{c}{ Values } \\
\hline $\begin{array}{l}\text { Maximum Affordable Delay ITU's } \\
\text { specification }\end{array}$ & \multicolumn{2}{c}{$3.0(\mathrm{~ms})+$ propagation time } \\
$\begin{array}{l}\text { Maximum Affordable Delay } \\
\text { for ring networks }\end{array}$ & \multicolumn{2}{c}{$3.0(\mathrm{~ms})+5(\mathrm{~ns} / \mathrm{m}) \times(\mathrm{N}-1) / 2 \mathrm{~L}(\mathrm{~km})$} \\
$\begin{array}{l}\text { Delay for accessing ring } \\
\text { Delay limit for echo cancellation }\end{array}$ & \multicolumn{2}{c}{$1.4(\mathrm{~ms})$} \\
Node processing time (through) & \multicolumn{2}{c}{$13.5(\mathrm{~ms})$} \\
Node Processing time (add/drop) & \multicolumn{2}{c}{$0.010(\mathrm{~ms})$} \\
\hline Longest route for restoration & \multicolumn{2}{c}{$0.005(\mathrm{~ms})$} \\
\cline { 2 - 4 } Path switching & Loopback switching \\
Node distance L & $(\mathrm{N}-1) \mathrm{L}(\mathrm{km})$ & $(3 \mathrm{~N}-5) / 2 \mathrm{~L}(\mathrm{~km})$ \\
Maximum number of nodes & $25(\mathrm{~km}) \quad 10(\mathrm{~km})$ & $25(\mathrm{~km}) \quad 10(\mathrm{~km})$ \\
\hline
\end{tabular}


$\operatorname{MAD}($ bi-ring $)=3.0(\mathrm{~ms})+5(\mathrm{~ns} / \mathrm{m}) \times(\mathrm{N}-1) / 2 \times \mathrm{L}(\mathrm{km})$,

where the propagation time per unit length in optical fiber is $5(\mathrm{~ns} / \mathrm{m})$.

3) Delay for network access is estimated by using the worst condition. The ADM may host an ATM-XC to support an ATM sub-network. Transmission bit-rate for the ATM subnetwork is $156 \mathrm{Mbit} / \mathrm{s}$, and the ATM-XC has 256 cell buffer (Aoyama, 1992). Buffer delay per cell is $2.7 \mu \mathrm{s} / \mathrm{cell}$ with the $156 \mathrm{Mbit} / \mathrm{s}$ line (Vickers, 1994). In the worst case, the buffers are full, therefore,

Access delay $=2.7(\mu \mathrm{s} /$ cell $) \times 256($ cell $) \times 2 \cong 1.4(\mathrm{~ms})$,

where, propagation time in the access cable is neglected. These conditions are summarized in Table 3, where processing time for passing-through and adding/dropping nodes are $\mathbf{0 . 0 1 0}$ $\mathrm{ms}$ and $0.005 \mathrm{~ms}$, respectively. Delay limit for implementing echo cancellation is $13.5 \mathrm{~ms}$ which equals the propagation distance specification $2,700 \mathrm{~km}$. The worst delay for the proposed path switching scheme is seen between nearest neighbor nodes i.e. ADM\#1 and ADM\#N. Therefore, in the proposed path switching scheme,

Protection delay $(\mathrm{PS})=1.4(\mathrm{~ms})+0.01(\mathrm{~ms}) \times(\mathrm{N}-1)+5(\mathrm{~ns} / \mathrm{m}) \times(\mathrm{N}-1) \times \mathrm{L}(\mathrm{km})$.

The worst case protection delay under loopback switching is seen on the path between nodes furthest from each other. The longest protection route is $(3 \mathrm{~N}-5) / 2 \times \mathrm{L}(\mathrm{km})$, therefore, the protection delay for loopback switching becomes,

Protection delay $(\mathrm{LB})=1.4(\mathrm{~ms})+0.01(\mathrm{~ms}) \times(3 \mathrm{~N}-1) / 2+5(\mathrm{~ns} / \mathrm{m}) \times(3 \mathrm{~N}-5) / 2 \times \mathrm{L}(\mathrm{km})$.

Figure 13 (a), (b) shows the estimation results, where $\mathrm{L}$ is $25 \mathrm{~km}$ in (a) and $10 \mathrm{~km}$ in (b). The architecture employing the proposed algorithm almost doubles the network scale than compared to loopback switching. Table 3 summarizes the network scale estimation considering delay. When the node distance is $25 \mathrm{~km}$, the proposed path switching scheme yields the total ring length of $575 \mathrm{~km}$, while loopback switching offers only $325 \mathrm{~km}$. With $10 \mathrm{~km}$ node distance, the ring lengths are 470 and $260 \mathrm{~km}$ for path switching and loopback switching, respectively.

\section{CONCLUSION}

This paper has studied a ring network architecture offering several tens of giga-bit/s that is applicable to future MANs or WANs. Each node connects sub-networks and LANs which provide widely differing bit-rates and protocols, and bundles these signals into a ring network using multiplexing techniques for high speed optical transmission systems such as SDH or WDM. Connectivity considerations for future services led us to the mesh connected logical topology wherein all ADMs are connected. This paper has proposed a novel bandwidth arrangement scheme for mesh-connected rings that minimizes the line capacity required. The scheme, which reuses bandwidths by converting byte positions/wavelengths, 
realizes a uniform add/drop procedure common to all nodes. The proposed scheme has several advantages. The first is that the simple and uniform procedure of each node is suitable for the hardware processing required in high-speed SDH or WDM networks, because tunable or programmable functions are not necessary. Second, this uniformity leads to cost reductions in equipment design and manufacture. Third, the proposed method is suitable for the distributed path-administration environment expected in future mesh-connected rings. Fourth, the scheme eliminates the bandwidth allocation problem. We also proposed a selfhealing algorithm suitable for the proposed ring that uses dual-ended shared path protection switching. The proposed swiching algorithm was compared with existing algorithms in SONET rings. The protection capacity required in the scheme is about $25 \%$ of that of SONET path switching. This algorithm results in shorter restoration paths than achieved by loopback switching, which yields shorter restoration times. Furthermore, it shows the best performance in terms of survivability under multiple failures. The network scale of the proposed ring is insensitive to the limitation caused by delay issues. Further studies are needed, for example the issue of network evolution: adding or deleting nodes, or interconnecting rings.

\section{ACKNOWLEDGEMENT}

The authors thank Kazuo Hagimoto, Kiyoshi Nakagawa, and Hideki Ishio for their encouragement. The authors also thank Yasuhiko Tada and Yoshiaki Sato for helpful discussions.

\section{APPENDIX}

A detail calculation of the restoration ratio as a function of restoration time is described against a cable cut.

\subsection{Restoration time for the proposed path switching}

With a cut fiber, the number of 'failure paths' is $\left(\mathrm{N}^{2}-1\right) / 8$. Restoration time for these paths consists of two kinds of delay. The first one is the guard time $T_{0}{ }^{1}$ for detecting P-ALM and SR, each of which is as long as 3 frames as in Automatic Protection Switching (APS) protocol. Second is the transmission delay for the restoration path $T_{0}^{2}$. Hence, restoration time $T$ is

$T=T_{0}^{1}+T_{0}^{2}$,

where, $T_{0}{ }^{l}$ is 0.75 (ms). The shortest path connected to the nearest neighbor node in the working line should take the longest route in the protection ring. Therefore, all paths are restored within the time taken to restore the shortest path. $T_{0}^{2}$ for the shortest path is $(N-1) t L+N T_{t h}+2 T_{a}$ where $t$ is the propagation time per unit length fiber, $L$ is the node distance, $T_{t h}$ and $T_{a}$ are processing times in pass-through node and add/drop node, respectively. Similarly, restoration time for the path connected to the second nearest neighbor is $T_{0}{ }^{l}+(N-2) t L+(N-1) T_{t h}+2 T_{a}$. Therefore, the restoration time for a path of arbitrary length $\mathrm{j}$ is 
$T=T_{0}^{1}+(N-j) t L+(N-j+1) T_{t h}+2 T_{a}$,

at which time longer paths than $\mathrm{j}$ are restored, where $1 \leq \mathrm{j} \leq(\mathrm{N}-1) / 2$. It should be noted that the path length equals the number of paths included in each link: there are $j$ paths of length $j$ in $a$ link. Therefore, the number of restored paths at $(a-2)$ is $\left(N^{2}-1\right) / 8-(j-1)(j-2) / 2$. Restoration ratio becomes,

$R(T)=\frac{N^{2}-1-4\left(N-1 \frac{T-\left(T_{0}^{1}+T_{t h}+2 T_{a}\right)}{t L+T_{t h}}\right)\left(N-2 \frac{T-\left(T_{0}^{1}+T_{t h}+2 T_{a}\right)}{t L+T_{t h}}\right)}{N^{2}-1}$.

\subsection{Restoration time for SONET loopback switching}

Restoration time for loopback switching consists of two kinds of delay as (a-1),

$T^{L}=T_{0}^{L 1}+T_{0}^{L 2}$.

$T_{0}^{L 1}$ is 0.75 (ms) which is double the 3 frame period (ITU, 1994). The remaining part $T_{0}^{L 2}$ is obtained as follows. Consider a path of arbitrary length $\mathrm{j}$ which traverses a failed cable in the working line. The failed link position on the path is assumed to be arbitrary $k: 1 \leq k \leq j$. Restoration path originating from the transmitting node approaches a node adjacent to the failure traveling through the working line. This distance is $(\mathrm{k}-1) \mathrm{L}$. At the node adjacent to the failure, the path is looped back to the protection ring, and then travels to the other node adjacent to the failure, so this protection line length is $(\mathrm{N}-1) \mathrm{L}$. The path then is loop backed to the working ring at the node. It then reaches the destination node having traversed the distance of $(\mathrm{j}-\mathrm{k}) \mathrm{L}$. Therefore, total distance of the restoration path is, $(\mathrm{k}-1) \mathrm{L}+(\mathrm{N}-1) \mathrm{L}+(\mathrm{j}-\mathrm{k}) \mathrm{L}=(\mathrm{N}+\mathrm{j}-2) \mathrm{L}$, which is independent of the failure position on path $\mathrm{k}$. Restoration time for the path of length $\mathrm{j}$ is,

$T=T_{0}^{1}+(N+j-2) t L+(N+j+1) T_{t h}+2 T_{a}$,

at which time the shorter paths than $\mathrm{j}$ are restored, because shorter paths have a shorter restoration time with loopback switching. The restoration ratio is,

$R(T)=\frac{4\left(N-1 \frac{T-\left(T_{0}^{L 1}+3 T_{t h}+2 T_{a}\right)}{t L+T_{t h}}\right)\left(N-2-\frac{T-\left(T_{0}^{L 1}+3 T_{t h}+2 T_{a}\right)}{t L+T_{t h}}\right)}{N^{2}-1}$.

\subsection{Restoration time for SONET path switching}

The calculation for SONET path switching is almost the same as for the proposed switching scheme except the guard time for switching request, and the number of failure paths in a cable. As in (a-1) or (a-4), restoration time is,

$T^{P}=T_{0}^{P 1}+T_{0}^{P 2}$, 
where $T_{0}{ }^{p 2}$ is the guard time just for failure detection, $0.375(\mathrm{~ms})$. A uni-directional ring has $\mathrm{N}(\mathrm{N}-1) / 2$ paths in a link. Therefore, the restoration ratio is,

$R(T)=\frac{N(N-1)-\left(N-1 \frac{T-\left(T_{0}^{P 1}+T_{t h}+2 T_{a}\right)}{t L+T_{t h}}\right)\left(N-2 \frac{T-\left(T_{0}^{P 1}+T_{t h}+2 T_{a}\right)}{t L+T_{t h}}\right)}{N(N-1)}$.

\section{REFERENCES}

Aoyama, T., Tokizawa, I., Sato, K. (1992) Introduction Strategy and Technologies for ATM VP-Based Broadband Networks: IEEE J. Select. Areas Commun., 10, No. 9, 1434-47.

Bellcore Technical Reference (1991) SONET Add/Drop Multiplexer Equipment (SONET ADM) Generic Criteria: Unidirectional, Dual Fed, Path Switched, Self-Healing Implementation: TR-TSY-000496, Supplement 1.

CCITT Recommendation G. 114 (1988) Mean One-way Propagation Time: Melbourne

Elrefaie, A. F. (1993) Multiwavelength Survivable Ring Network Architectures: IEEE ICC '93, 1245-51.

Fischer, W., Wallmeier, E., Worster, E., Davis, S. P., and Hayter, A. (1994) Data Communications Using ATM: Architectures, Protocols, and Resource Management: IEEE Commun. Mag., 32, No.8, 24-33.

Hill, G. R. (1988) A Wavelength Routing Approach to Optical Communications Network: INFOCOM ' 88, 354-62.

Hill, G.R., Chidgey, P. J., Kaufhold, F., Lynch, T., Sahlen, O., Custavsson, M., Janson, M., Lagerstrom, B., Grasso, G., Meli, F., Johansson, S., Ingers, J., Fernandez, L., Rotolo, S., Antonielli, A., Tebaldini, S., Vezzoni, E., Caddedu, R., Caponio, N., Testa, F., Scavennec, A., O'Mahony, M. J., Zhou, J., Yu, A., Sohler, W., Rust, U., and Herrmann, H. (1993) A Transport Network Layer Based on Optical Network Elements: J. Lightwave Technol., 11, No. 5/6, 667-79.

ITU-Telecommunication Standardization Sector (1994) Draft Recommendation G. SHR-1: Study Group 15, Geneva, May.

Kataoka, T., Miyamoto, Y., Hagimoto, K., Wakita, K., and Kotaka, I. (1992) Ultrahighspeed Driverless MQW Intensity Modulator and $20 \mathrm{Gbit} / \mathrm{s}, 100 \mathrm{~km}$ Transmission Experiments: Electron. Lett., 28, No. 10, 867-8.

Kawanishi, S., Takara, H., Uchiyama, K., Kitoh, T., and Saruwatari, M. (1993) $100 \mathrm{Gbit} / \mathrm{s}$, $50 \mathrm{~km}$ and Non-repeated Optical Transmission Employing All-Optical MultiDemultiplexing and PLL Timing Extraction: Electron. Lett., 29, No. 12, 1075-6.

Kobayashi, Y., Sato, Y., Aida, K., Hagimoto, K., and Nakagawa, K. (1994) SDH-Based 10 Gbit/s Optical Transmission Systems: IEEE GLOBECOM '94, 1166-70.

Liew, S. C. and Lu, K. W. (1994) A Framework for Characterizing Disaster-Based Network Survivability: IEEE J. Select. Areas Commun., 12, No. 1, 52-8.

McEachern, J. A. (1992) Gigabit Networking on the Public Transmission Network: IEEE Commun. Mag., April, 70-8. 
Nosu, K., Toba, H., Inoue, K., and Oda, K. (1993) 100 Channel Optical FDM Technology and its Applications to Optical FDM Channel Based Networks: J. Lightwave Technol., 11, No.5/6, 764-76.

Partridge, C. (1994) Gigabit Networking: Addison-Wesley, Massachusetts.

Sato, K., Okamoto, S., and Hadama, H. (1994) Network Performance and Integrity Enhancement with Optical Path Layer Technologies: IEEE J. Select. Areas Commun., 12, No.1, 159-70.

To, M. and McEachern, J. A. (1991) Planning and Deploying a SONET-based Metro Network: IEEE LTS, Nov., 19-23.

Tomizawa, M., Yamabayashi, Y., Kawase, N., and Kobayashi, Y. (1994) Self-Healing Algorithm for Logical Mesh Connection on Ring Networks: Electron. Lett., 30, No. 19, 1614-5.

Tomizawa, M., Yamabayashi, Y., Sato, Y., Kobayashi, Y., and Nakagawa, K., to be submitted.

Vickers, B. J. and Suda, T. (1994) Connectionless Srevices for Public ATM Networks: IEEE Commun. Mag., 32, No. 8, 34-42.

Wasem, O., Wu, T.-H., and Cardwell, R.-H. (1992) OC-96/OC-192 Technology Impact on SONET Self-Healing Rings: Conf. OFC'92, 257.

Wu, T.-H. and Lau, R.-C. (1990) A Class of Self-Healing Ring Architectures for SONET Network Applications: IEEE GLOBECOM'90, 444-51.

Wu, T.-H. (1992) Fiber Network Survivability: Artech House, Boston and London.

Yamabayashi, Y., Matsuoka, S., Uematsu, Y., Hohkawa, K., and Ohata, M. (1993) Line Terminating Multiplexers for SDH Optical Networks: J. Lightwave Technol., 11, No. 5/ 6. 875-81.

\section{AUTHORS' BIOGRAPHIES}

Masahito Tomizawa received the B.E. degree in applied physics in 1990 and the M.Sc. degree in physics in 1992 from Waseda University (Tokyo). In 1992, he joined NTT Transmission Systems Laboratories. He is currently engaged in research on high-speed optical network architectures and implementing equipments.

Mr. Tomizawa is a member of the Institute of Electrical and Electronics Engineers, and of the Institute of Electronics, Information and Communications Engineers, and Physical Society of Japan.

Yoshiaki Yamabayashi received the B.E. and the M.E. degree in applied physics form Hokkaido University in 1979 and 1981, respectively. In 1981, he joined NTT Electrical Communication Laboratories. He has been engaged in research and development on Line terminating multiplexer in $10 \mathrm{Gbit} / \mathrm{s}$ optical transmission systems.

Mr. Yamabayashi is a member of the Institute of Electrical and Electronics Engineers, the Optical Society of America, the Japanese Society of Applied Physics, and the Electronics, Information and Communications Engineers of Japan. 
Nobuyuki Kawase was born in Niigata, Japan, on May 13, 1961. In 1993, he joined NTT Transmission Systems Laboratories. He has been engaged in development on $10 \mathrm{Gbit} / \mathrm{s}$ Line terminating multiplexer, and is currently engaged in research and development on algorithm and equipment of hitless path switching scheme in SDH systems.

Mr. Kawase is a member of the Institute of Electronics, Information and Communications Engineers of Japan.

Yukio Kobayashi received the B.E. and the M.E in electrical engineering from Nagoya University in 1971 and 1973, respectively. He joined NTT Electrical Communication Laboratories in 1973, and he has been engaged in research and development on undersea and trunk transmission systems. He is presently working on $10 \mathrm{Gbit} / \mathrm{s}$ optical transmission systems. He is currently a Senior Research Engineer, Supervisor in Lightwave Communications Laboratory of NTT.

Mr. Kobayashi is a member of the Institute of Electronics, Information and Communications Engineers of Japan. 